\title{
P46 Haemodynamics Determinants of Central Pressure during Systole
}

\author{
Samuel Vennin*, Ye Li, Jorge Mariscal-Harana, Haotian Gu, Henry Fok, Jordi Alastruey, Phil Chowienczyk
}

King's College London, UK

\section{ABSTRACT}

The influence of arterial and ventricular parameters on the main fiducial pressure points and index during systole has been investigated using a mix of in silico and in vivo data. Notably, an index, QIx, based entirely on ventricular ejection patterns has been developed and its potential in describing the augmentation pressure index, AIx, has been investigated and compared against the reflection coefficient $\Gamma$. In a group of hypertensive patients $(n=156)$ and in healthy volunteers whose physiology was modulated via administration of vasoactive drugs $(n=13)$, QIx was found to be more correlated to AIx than $\Gamma$ (coefficient of determination, $R^{2}=0.71$ vs $R^{2}=0.53$ in the normotensive group; $R^{2}=0.52$ vs $R^{2}=0.37$ in the hypertensive group). This trend was confirmed by a LASSO analysis on in vivo data (standardised coefficient, $\beta=0.42 \mathrm{vs} \beta=0.22, p<0.001$ in both cases) and also observed in an in silicosensitivity analysis on a reduced 7-artery model of the upper aortic arch (for variations between $-25 \%$ and $+25 \%$ of QIx and $\Gamma$ from baseline values, AIx varied between $-245 \%$ and $+193 \%,-194 \%$ and $+191 \%$ respectively) (Figure 1 ). All analyses were also concordant for the main determinants of the first systolic shoulder P1 and augmentation pressure AP: pulse wave velocity $(\beta=0.74, p<0.001)$ and peak flow velocity $(\beta=0.41, p<0.001)$ are the main determinants of P1 while reflection waves $(\beta=0.34, p<0.001)$ and stroke volume $(\beta=0.37, p<0.001)$ mainly define AP (Figure 1$)$. These results further strengthen the case that ventricular dynamics is at least as important as arterial stiffening in raised pulse pressure.
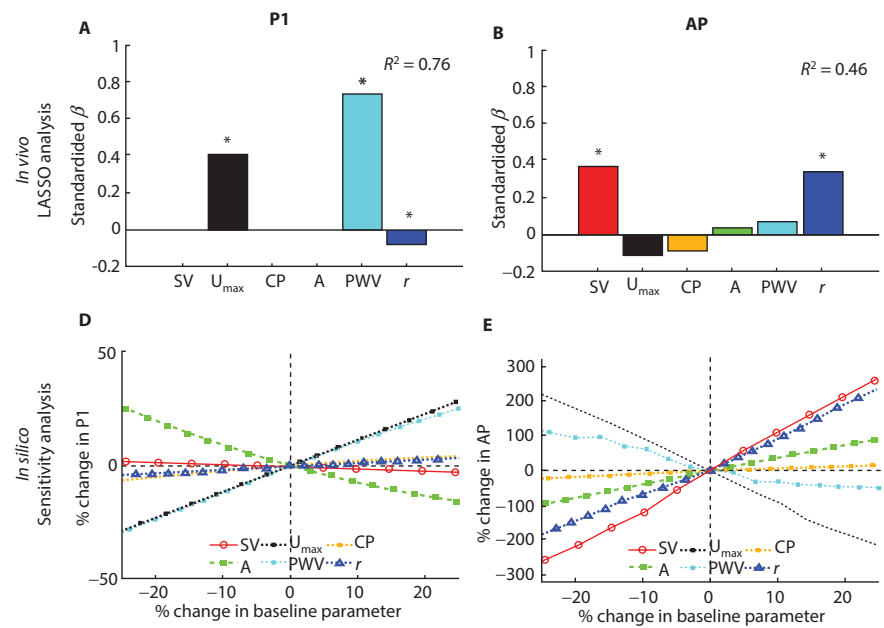

$$
\text { C }
$$
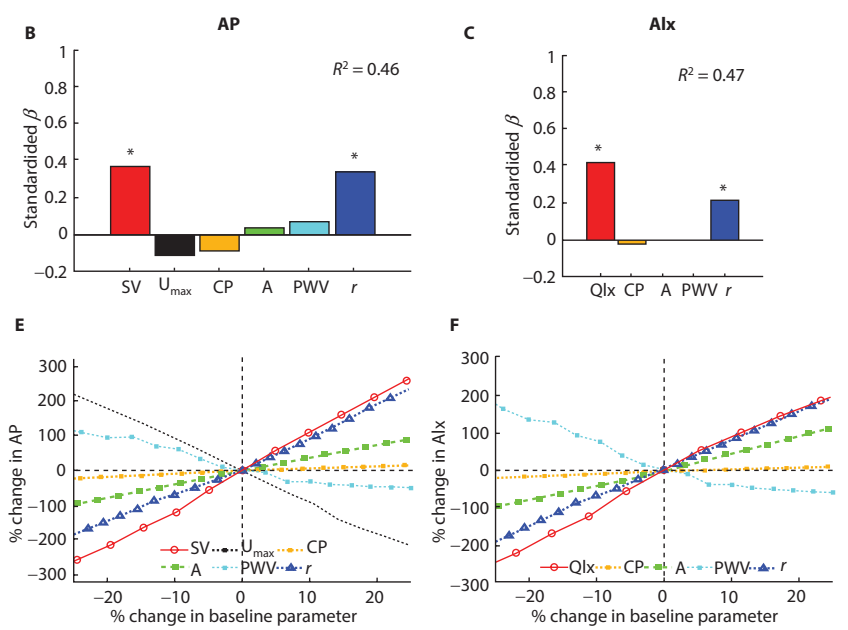

Figure 1

(c) 2019 Association for Research into Arterial Structure and Physiology. Publishing services by Atlantis Press International B.V. This is an open access article distributed under the CC BY-NC 4.0 license (http://creativecommons.org/licenses/by-nc/4.0/). 